\title{
Heart rate reactivity, habituation, and associative learning in developmentally disabled preschool children
}

\author{
D. S. TUBER, A. E. RONCA, G. G. BERNTSON, S. T. BOYSEN, and H. LELAND \\ Ohio State University, Columbus, Ohio
}

\begin{abstract}
The relationship between adaptive behavior and cardiac indices of orienting, habituation, and associative learning were evaluated in a heterogeneous population of developmentally disabled infants and young children. Cardiac reactivity and habituation were examined through responses to simple nonsignal stimuli, and associative learning was evaluated by the cardiac response to stimulus omission in a conditioned expectancy paradigm. Results revealed a significant negative correlation between the magnitude of the deceleratory orienting response and adaptive competence, as measured by the Adaptive Behavior Scale for Infants and Early Childhood. This correlation was independent of both age and baseline heart rate. In contrast, habituation and simple associative learning were not related to the level of adaptive behavior. These findings suggest that cardiac reactivity may provide a sensitive measure for the evaluation of functional capacities of impaired populations.
\end{abstract}

The psychophysiological correlates of behavioral competence in the developmentally disabled infant and young child have received limited experimental attention. Impediments related to maturational status and physical impairment often preclude the application of conventional testing paradigms to these children. In this regard, psychophysiological approaches that do not require the active participation of the subject may be useful in characterizing the functional deficits of this population. Such an approach has proven useful in identifying cognitive features and diagnostic markers in high-risk, organically retarded, and severely brain-damaged infants (Berntson, Tuber, Ronca, \& Bachman, 1983; Bradley-Johnson \& Travers, 1979; Fox \& Lewis, 1983; Krafchuk, Tronick, $\&$ Clifton, 1983; Porges, 1983; Tuber, Berntson, Bachman, \& Allen, 1980).

Although autonomic measures have been applied in previous studies of older developmentally disabled children and adolescents, conclusions of appreciable generality or specificity remain difficult to derive from this literature (Karrer, Nelson, \& Galbraith, 1979; Stern \& Janes, 1973). Accordingly, cardiac measures of the orienting response have been suggested for use as indicators of overreactivity, underreactivity, or normal reactivity in mentally retarded subjects (Johnson, 1976; Karrer et al., 1979). It is increasingly apparent, however, that a host

\footnotetext{
This research was supported in part by NIH Grant HD15865. We thank Lisa Barclay for the collection of ABSI data, and Sherrie Ireland and the staff of the Franklin County MRDD program for their cooperation. Portions of these data have been presented in brief form (American Journal of Mental Deficiency, January 1985). Address correspondence to D. S. Tuber, 48 Townshend Hall, Ohio State University, Columbus, OH 43210-1222.
}

of variables can contribute to the pattern of cardiac response, including the characteristics of the experimental stimuli, their signal value, and the context in which they are delivered (Johnson, 1976; Karrer et al., 1979). Moreover, features of the subject populations assume special significance, and differences in functional characteristics of subject groups can dramatically influence the pattern of results and render comparisons among studies difficult (Karrer et al., 1979). One approach to these problems is to restrict analysis to specific subpopulations which manifest common developmental anomalies, an approach that has yielded differential patterns of results among groups of retarded subjects (Clausen, Lidsky, \& Serson, 1976). Such diagnostic categories, however, are generally based on etiological or neurological, rather than functional, considerations, and the psychophysiological results may relate more directly to differences in the functional abilities of the subjects (Johnson, 1976; Vogel, 1961). Moreover, diagnostic classifications often do not adequately reflect the broad individual variability in behavioral competence exhibited by individuals within these groups (Leland, 1982). An alternate approach is to utilize an index of functional ability as a dimension along which to explore psychophysiological relationships. Although IQ has been employed as such an index (Johnson, 1976; Vogel, 1961), its applicability to young and often severely impaired children is limited.

In view of these considerations, we have employed standard paradigms to examine patterns of cardiac reactivity, habituation, and associative learning in a heterogeneous population of developmentally disabled infants and preschool children, who vary along dimensions of behavioral competence. For each subject, a measure of adaptive behavior was available to serve as a basis for explor- 
ing psycophysiological relationships. Reactivity and habituation were examined through cardiac responses to three modalities of stimuli, and associative learning was evaluated by the cardiac response to stimulus omission in a conditioned expectancy paradigm.

\section{METHOD}

\section{Subjects}

The subjects were 33 noninstitutionalized children from the Franklin County (Ohio) Mental Retardation/Developmental Disabilities preschool program. Characteristics of the subject population are outlined in Table 1 . The subjects were classified into three conventional diagnostic categories (developmental delay, trisomy-21, and cerebral palsy), and a fourth miscellaneous group which included microcephaly, cerebral atrophy, hydrocephalus, and undiagnosed conditions. Trisomy- 21 was, in all cases, identified by karyotype. Subjects in the cerebral palsy group were relatively homogeneous in that all were severely spastic, nonambulatory, and alinguistic. The subjects ranged in age from 1 to 6 years, with the mean ages of the four groups being comparable [ANOVA, $F(3,29)$ $=.69]$.

\section{Assessment}

The level of adaptive behavioral function was evaluated for each subject by the Adaptive Behavior Scale for Infants and Early Childhood (ABSI), an extension of the AAMD Adaptive Behavior Scale (Leland, Shoaee, McElwain, \& Christie, 1981; Nihira, Foster, Shellhaas, \& Leland, 1974). The scale, consisting of 63 items which tabulate the adaptive behaviors displayed by the child, is essentially an inventory of skills in seven domains of adaptive functioning (Independent Functioning, Physical Development, Communication Skills, Concept Skills, Play, Self-directed Behavior, and Personal Responsibility and Socialization). Based on an interview with the parent or teacher, the child is scored for level of proficiency on the various skills. (In order to maintain consistency in the present study, all ABSI data were derived from an interview with teachers of the Franklin County Program.) The accumulated score for each of the domains provides a profile of behavioral competency with minimal theoretical structuring of the data (Leland et al., 1981). The seven domains have high reliability (test-retest over 2 weeks, $r=.81$ to .94$)$. In the present study, the obtained ABSI scores were highly correlated $(r=.93)$ with a first-party, objective assessment of adaptive behavior (Preschool Developmental Profile; D'Eugenio \& Moersch, 1981), which was available for 20 of the 33 subjects.

For the present study, the total raw score on the seven domains of the ABSI served as an overall index of adaptive behavior, and age-normalized scores on the individual domains were used for subtest analyses. As illustrated in Table 1, significant group differences

Table 1

Subject Population

\begin{tabular}{|c|c|c|c|c|c|}
\hline Group & $\mathrm{N}$ & Age* & $\begin{array}{c}\text { Baseline } \\
\text { Heart Rate } \dagger\end{array}$ & $\begin{array}{l}\text { ABSI } \\
\text { Score } \ddagger\end{array}$ & $\begin{array}{l}\text { ABSI } \\
\text { Norm§ }\end{array}$ \\
\hline $\begin{array}{l}\text { Developmental } \\
\text { Delay }\end{array}$ & 12 & $4.2 / 1.1$ & 110.5 & $181.2 / 59.4$ & 276.4 \\
\hline $\begin{array}{l}\text { Trisomy- } \\
21\end{array}$ & 8 & $3.8 / 1.8$ & 117.5 & $186.2 / 70.0$ & 253.7 \\
\hline $\begin{array}{c}\text { Cerebral } \\
\text { Palsy }\end{array}$ & 6 & $3.2 / 1.6$ & 115.5 & $62.6 / 59.3$ & 253.8 \\
\hline Miscellaneous & 7 & $4.1 / 1.6$ & 108.9 & $125.8 / 78.5$ & 277.1 \\
\hline
\end{tabular}

*Mean and standard deviation of age in years. $†$ Mean baseline heart rate (bpm) derived from inventory session. $\quad \ddagger$ Mean and standard deviation of ABSI score. \$Mean ABSI score from age-matched norm groups derived from Leland et al. (1981). were apparent in overall ABSI scores [ANOVA, $\mathrm{F}(3,29)=5.56$, $\mathrm{p}=.004]$, although substantial variability was also apparent within the groups, as indicated by the large standard deviations.

\section{Procedure}

Sensory inventory session. Basic cardiac reactivity was evaluated in the first of three test sessions, each separated by a minimum of 1 day. In this session, subjects received six presentations of moderate-intensity nonsignal stimuli of three modalities: a tone (1-kHz square wave at $76 \mathrm{~dB}$ SPL, $<10-\mathrm{msec}$ rise time, delivered via a free-field speaker), a diffused light $\left(1,000 \mathrm{~lm} / \mathrm{m}^{2}\right.$ at the subject plane), and a vibrotactile stimulus $(0.5-\mathrm{mm}$ displacement at $18 \mathrm{~Hz}$ provided by a small motor with an eccentric cam affixed to the right gastrocnemius with velcro straps). All stimuli were $1 \mathrm{sec}$ in duration, with the six stimuli of a given sensory modality being presented in successive trials (randomized 25-45-sec intertrial interval. The three modalities were tested in fixed order (tone, light, vibrator), to maximize comparability of the treatments and permit regression analyses across the heterogeneous subject population. Testing was accomplished in a quiet room (60-dB white noise), with the subject seated comfortably in a bean-bag chair. Prior to this and subsequent sessions, special care was taken to familiarize the subject with the experimental environment, and to provide a pleasant and friendly testing context. To further minimize apprehension and to monitor the subject, an experimenter sat beside, but did not interact with, the child during testing.

Conditioned expectancy sessions. Associative learning was evaluated in the two subsequent sessions through the use of a modified conditioned expectancy or omission paradigm, which allows the assessment of conditioned associations between innocuous stimuli (Grings, 1960; Sokolov, 1963). Two identical tone stimuli, having a fixed temporal relationship, were repeatedly presented to the subjects in conditioning trials. Interspersed within these conditioning trials were occasional test trials in which the first stimulus was presented alone. Characteristically, such conditioning results in the development of an association between the stimuli, such that the first stimulus becomes a signal for the occurrence of the subsequent stimulus. To the extent that the first stimulus acquires this signal value, the unexpected omission of the second stimulus in test trials introduces a disparity, which is detectable through a variety of somatic and autonomic indices of the orienting response (Grings, 1960; Sokolov, 1963). Each subject received two sessions with the same tone stimulus used during the Inventory phase. Prior to each session, the subjects were given a single presentation of the tone to obtain an index of the response to the test stimulus prior to conditioning. The paired tone stimuli were then repeatedly presented in conditioning trials $(1,500$-msec interstimulus interval, 500-msec trace), and a single-tone stimulus was presented in interspersed test trials. Intertrial intervals averaged $35 \mathrm{sec}(25$ to $45 \mathrm{sec}$, block randomized). A total of 28 conditioning trials and 4 test trials (Trials $10,17,26$, and 34 ) were given in each session.

\section{Data Recording}

Silver/silver chloride EKG electrodes were attached to monitor sites V6, A1, and A2. The EKG signal was amplified by an Amerec ERM 101 cardiotachometer and recorded, along with a stimulus marker, on a Burdick FM cardiocassette recorder. The R-wave pulse output from the cardiotachometer was coupled to a microprocessor data-acquisition system for the on-line determination of individual heart periods (1-msec resolution). The FM tape was subsequently played back on a Grass Model 7 polygraph $(30 \mathrm{~mm} / \mathrm{sec})$ for a permanent record and for artifact editing.

\section{Data Reduction and Analysis}

Potential artifacts in the heart period data were identified off-line by the microcomputer system as periods that deviated from either of the immediately surrounding beats by more than $30 \%$. Heart periods exceeding this criteria were checked, and corrected if neces- 
sary, by direct measurement from the polygraph record. Individual heart periods were then assigned to $1-\mathrm{sec}$ peristimulus time bins, averaged, and converted to rate measures. Individual trials in which the mean or standard deviation of the prestimulus heart rate exceeded the corresponding session values by more than $2 \mathrm{SD}$, or in which a systematic trend in heart rate was apparent, were eliminated from analysis. Collectively, these criteria resulted in the elimination of less than $6 \%$ of the trials.

Trends analyses over one prestimulus and three poststimulus 5sec time blocks were used for descriptive characterization of the patterns of cardiac response and for statistical evaluations of differences in response components across conditions. In addition, indices of the magnitude of cardiac responses were derived as the mean change in heart rate from the 5-sec prestimulus baseline to the 10 -sec period following the stimulus. Statistical evaluations of response magnitudes across conditions were based on a betweensubjects analysis of variance, with a covariate on baseline heart rate. Finally, multiple regression analyses were used to explore the relationships between psychophysiological response dimensions and ABSI scores.

To obtain an estimate of habituation that was relatively independent of heart rate lability, an index was derived in which the change in the magnitude of the response over trials was scaled in proportion to the overall heart rate lability. This habituation index is as follows:

$$
\mathbf{H}=|\mathbf{R a}-\mathbf{R b}| /(|\mathbf{R a}|+|\mathbf{R b}|),
$$

where $\mathrm{H}$ is the habituation index, $\mathrm{Ra}$ is the signed magnitude of the initial cardiac response, and $\mathrm{Rb}$ is the signed magnitude of the final response. This index can range from +1 (habituation) to -1 (sensitization).

\section{RESULTS}

\section{Sensory Inventory}

Descriptive data. Cardiac responses to all modalities of stimuli in the initial inventory sessions were predominately deceleratory for all subject groups, as illustrated in Figure 1A. An analysis of trends revealed a significant cardiac response to the stimuli $[F(3,96)=$ $12.26, \mathrm{p}<.001]$, characterized by significant quadratic

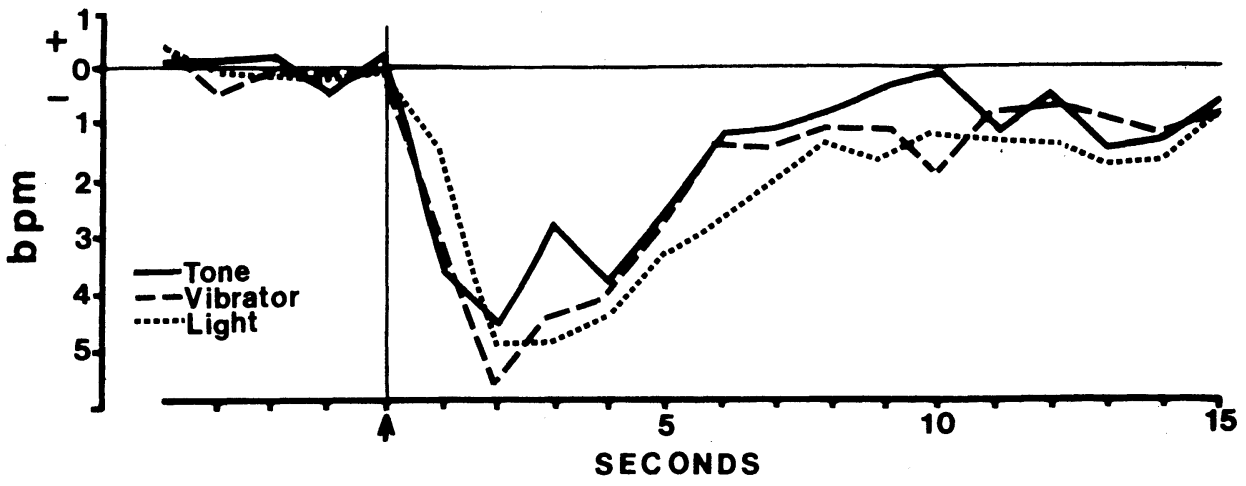

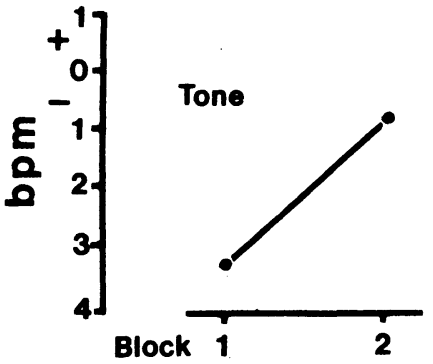
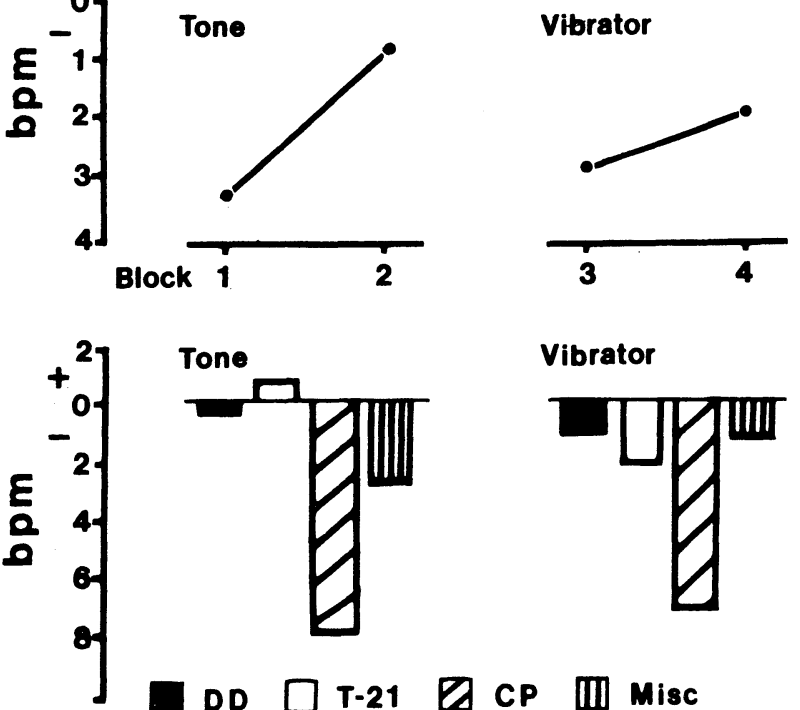

DD

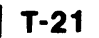

Vibrator

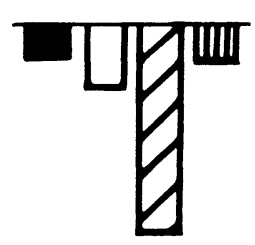

Light
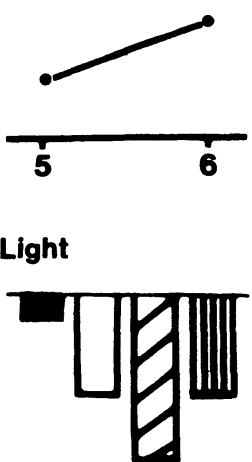

Figure 1. (A) Heart rate responses to nonsignal stimuli (six trials of each), averaged over all subjects. Responses are plotted from prestimulus baseline, with the time of stimulus presentation indicated by the arrow. (B) Magnitude of the cardiac responses (for the 10-msec poststimulus period) over two three-trial blocks. (C) Magnitude of the overall cardiac responses by subject group. 
$[\mathrm{F}(1,32)=14.53, \mathrm{p}<.001]$ and cubic components $[F(1,32)=13.61, p<.004]$. No main effect of modality emerged, nor was there a significant interaction between modality and any orthogonal response component. To examine potential group differences in the magnitude of the cardiac responses, the data were subjected to a repeated measures analysis of variance on the heart rate response for the 10 -sec period after the stimulus, according to a 4 (groups) $\times 3$ (modalities) design, with a covariate on baseline heart rate. This analysis revealed significant differences in the magnitude of the response across subject groups $[F(3,28)=6.04, p<.002]$, with the $C P$ group showing the largest decelerations and the DD group the smallest (Figure 1C). There was no significant main effect of modality or group $\times$ modality interaction, and no significant covariation with baseline heart rate emerged.

The cardiac responses to all stimuli were subject to habituation over trials, as is illustrated in Figure 1B. Analysis of variance on the index of habituation (from the first three to the last three trials; see Methods) revealed no significant differences across subject groups $[F(3,29)=.11]$ or across stimulus modalities $[\mathrm{F}(2,58)=.26]$. Nor did a significant group $\times$ modality interaction emerge $[\mathrm{F}(6,58)=1.5]$.

Regression analysis. Although subjects were divided into conventional diagnostic categories for the descriptive analyses outlined above, our primary interest was in the relationship between cardiac response patterns and adaptive behaviors. Consequently, the present data were subjected to multiple regression analysis. Scores from the ABSI served as the criterion, with the following as predictor variables: age, baseline heart rate, cardiac response magnitudes for each stimulus modality, and habituation indices for the three stimuli. This analysis revealed that approximately two-thirds of the variance in ABSI scores could be accounted for by the predictor variables $[R=$ $.66 ; \mathrm{F}(8,24)=5.86, \mathrm{p}<.001$ ], with age and cardiac reactivity emerging as independent and strongly predictive factors. This predictive relationship held for subjects in all diagnostic categories, and no significant differences existed across groups in the residual error of the predicted ABSI scores $[\mathrm{F}(3,29)=1.97$; Figure 2].

As expected, age emerged as a significant predictor of ABSI level $[\mathrm{r}=.35$; partial correlation $=.55 ; \mathrm{F}(1,24)$ $=10.4, p<.005]$. By far the most predictive variable, however, was cardiac reactivity to the vibrotactile stimulus, with large decelerative responses associated with low ABSI scores $[r=.65 ;$ partial correlation $=.61 ; F(1,24)$ $=14.3, p<.001]$. The high partial correlations of age and of reactivity to the vibrotactile stimulus, and their related lack of intercorrelation $(r=-.01)$, indicate that age and reactivity account for different aspects of the ABSI variance, and provide unique contributions to the predictive relationship. No other predictor variable achieved significance in the regression analysis, although the independent correlations between ABSI levels and the cardiac response magnitudes to the tone and the light were significant $(r=.37$ and $.41 ; \mathrm{ps}<.05)$. The responses to these stimuli, however, were intercorrelated with reactivity to the vibrotactile stimulus $(r=.35$ and .52$)$, and their partial correlations with ABSI scores were low (rs $<.10)$. The correlations between ABSI level and the indices of habituation were uniformly low and insignificant (rs < .30; partial correlations < .04). Baseline heart rate, while negatively correlated with age $(r=-.52 ; p<.05)$, was uncorrelated with ABSI score $(r=-.05)$ or with cardiac reactivity measures ( $\mathrm{rs}=.00$ to -.31 ).

The correlation between cardiac reactivity and ABSI scores did not appear to be specific to a particular dimension of adaptive behavior. Significant correlations were found between cardiac reactivity to the vibrotactile stimulus and each of the individual subscales of the ABSI (Independent Functioning, $r=.64$; Physical Development, $\mathrm{r}=.59$; Communications Skills, $\mathrm{r}=.62$; Concept Skills, $r=.45$; Play, $r=.59$; Self-directed Behavior, $r=.47$; Personal Responsibility and Socialization, $r=.59$ ).

Drug treatments can seriously confound psychophysiological studies, and 12 of the 33 subjects of the present study were on some form of medication. In most cases (10), this entailed a daily regimen of pentobarbital (30$90 \mathrm{mg} /$ day). To evaluate the potential relationship between drug treatment and cardiac reactivity, we examined the correlation between pentobarbital dose and the magnitude of the cardiac response. This correlation was small and did not achieve significance, either when considered over all subjects $(r=-.26)$ or when limited to the pentobarbital subgroup $(r=.18)$. These findings suggest that

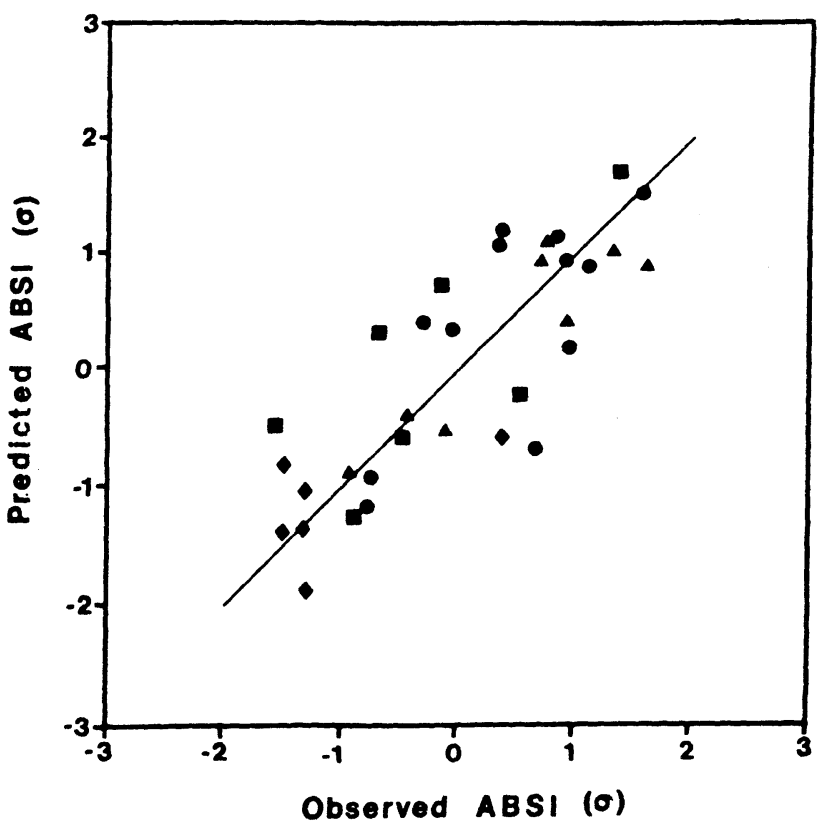

Figure 2. Observed ABSI scores plotted against those predicted by regression analysis. (Circles = DD group, triangles = TR group, diamonds $=\mathrm{CP}$ group, and squares $=\mathrm{MI}$ group.) 
drug regimen introduced minimal confounding of cardiac response measures in the present study.

\section{Conditioned Expectancy Paradigm}

The results outlined above indicate that the magnitude of the deceleratory cardiac orienting response is highly predictive of the level of adaptive behavioral function across a broad range of developmentally disabled infants and young children. Moreover, this reactivity is largely independent of age, and accounts for a distinct aspect of the ABSI variance. In contrast, habituation and baseline heart rate were largely unpredictive of the level of adaptive functioning. To further examine these relationships and to explore the predictive utility of a measure of associative learning, all subjects were given additional testing in a conditioned expectancy paradigm. For these sessions, cardiac responses were obtained to paired tone stimuli on conditioning trials and to a single tone on interspersed test trials in which the second stimulus was unexpectedly omitted.

Descriptive data. The cardiac responses to the presentation of the single-tone stimulus prior to stimulus pairings were virtually identical to the deceleratory responses observed to the initial presentation of the identical tone in the inventory sessions (mean response $=-4.6 \mathrm{bpm}$ in the inventory session and $-4.7 \mathrm{bpm}$ in the conditioning sessions). Cardiac responses to the paired tone stimuli in the conditioning trials were also deceleratory. Consistent with the results from the inventory session, analysis of variance revealed significant differences in the magnitude of the cardiac response over the first six conditioning trials, with the CP subjects again showing the largest responses and the DD group the smallest [mean response for the DD group $=-.4 \mathrm{bpm}, \mathrm{TR}$ group $=-2.7, \mathrm{CP}$ group $=-6.2$, and the MI group $=-1.8 ; \mathrm{F}(3,29)=$ $5.61, \mathrm{p}<.005]$.

Habituation to the paired tone stimuli appeared to be slower than to the single stimuli in the inventory sessions, and cardiac response magnitudes did not differ over the first nine trials (mean responses for blocks of three trials $=-2.2,-2.4$, and $-2.3 \mathrm{bpm}$ ). Habituation was evidenced on subsequent trials, however, as is apparent by the responses on the later conditioning trials which immediately preceded the test trials throughout the sessions (Figure 3). An index of habituation (see Methods) was derived for the change in response from the first three to the last three conditioning trials of each session. Consistent with the findings outlined above, no significant differences existed in the degree of habituation across subject groups $[F(3,29)=1.77]$.

After conditioning, the omission of the second stimulus on test trials resulted in a reinstatement or enhancement of the deceleratory cardiac orienting response (Figure 3). The response to stimulus omission differed considerably from the prevailing response to the paired conditioning stimuli, and from the response to the single stimulus alone prior to conditioning. While an initial deceleration was observed in all cases, the response on test trials demonstrated a marked prolongation of this deceleration, consistent with the appearance of an OR to the omission of the second stimulus. An analysis of trends on the heart rate data revealed a significant main effect of time blocks $[F(3,96)=5.38, p<.001]$, revealing the stimulus-induced changes in heart rate. A significant timeblock $\times$ test/control condition interaction $[F(3,96)=$ $8.81, \mathrm{p}<.001]$ reflected the selective long-lasting decelerative component on the test trials. This interaction was attributable to significant differences in the linear trend component between the test and control trials $[F(1,32)=15.91, p<.001]$. No significant group differences were apparent in the magnitude of the omission response [response on control trials minus the response on test trials; $F(3,29)=1.73$ ].

Regression analysis. The data from the conditioned expectancy paradigm were submitted to a regression analysis, with ABSI score serving as the criterion and the following as predictor variables: age, baseline heart rate, magnitude of the cardiac response to the paired stimuli, the index of habituation to the paired stimuli, and the magnitude of the omission response (as defined above). Results again revealed a significant relationship between ABSI scores and the predictor variables $[R=.36 ; F(5,27)=$ $3.10, p<.02]$. Consistent with the findings from the inventory sessions, the magnitude of cardiac reactivity to the conditioning stimuli was significantly correlated with ABSI scores $[\mathrm{r}=.52$, partial correlation $=.47 ; \mathrm{F}(1,27)$ $=7.78, \mathrm{p}<.01]$. No other predictor variable achieved significance in the regression analysis (habituation, $r=$ .16 , partial correlation $=.03$; response to stimulus omission, $\mathrm{r}=.18$, partial correlation $=.03$; baseline heart rate, $\mathrm{r}=-.01$, partial correlation $=-.31$ ).

Age was again negatively correlated with baseline heart rate $(\mathrm{r}=-.49 ; \mathrm{p}<.01)$. Moreover, although age showed a significant independent correlation with ABSI scores $(\mathrm{r}=.35 ; \mathrm{p}<.05)$, its partial correlation was low (.27) and failed to achieve significance $[\mathrm{F}(1,27)=2.2]$. This was related, in part, to the fact that age was significantly intercorrelated with cardiac reactivity to the paired stimuli $(\mathrm{r}=.45 ; \mathrm{p}<.02)$, and consequently did not provide a unique predictive contribution to the regression analysis. These findings are in contrast to the results from the inventory sessions, in which age and cardiac reactivity to the single stimuli were uncorrelated. Moreover, responses to the single-tone stimulus presented prior to stimulus pairings in the conditioning sessions, although correlated with ABSI level $(r=.35 ; \mathrm{p}<.05)$, were also uncorrelated with age $(\mathrm{r}=-.03)$. The intercorrelation between age and cardiac reactivity to the paired conditioning stimuli is probably attributable to the obtained association between age and baseline heart rate, and to the relationship between cardiac response magnitude and baseline $(\mathrm{r}=.35 ; \mathrm{p}<.05)$, which selectively appeared with the stimulus pairings. It remains unclear whether this latter relationship emerged as a result of stimulus pairing, per se, or to some other dimension of the stimulus complex. 


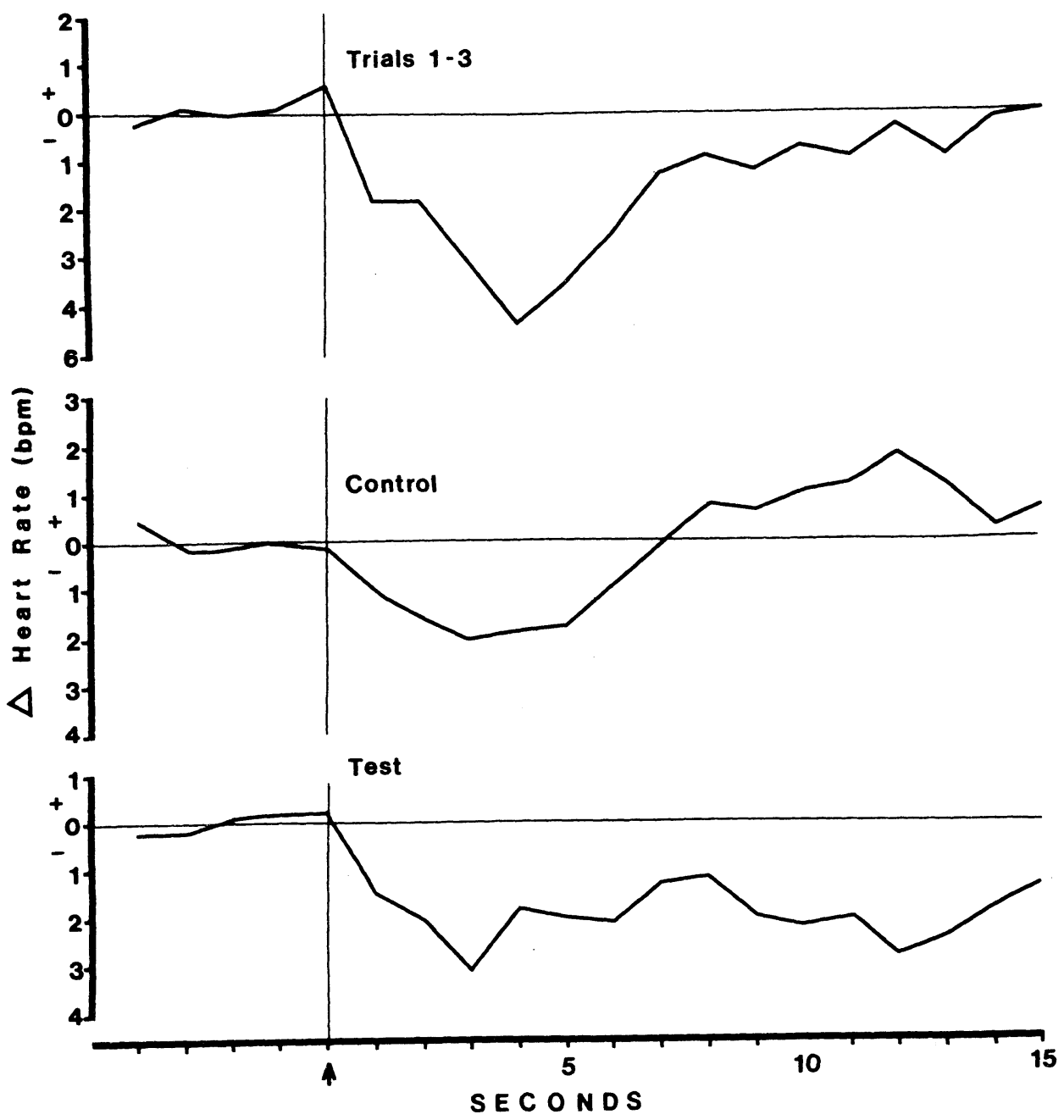

Figure 3. Heart rate responses in the conditioned expectancy sessions. Top: Mean response to the single-tone stimulus prior to conditioning. Middle: Response to the paired conditioning stimuli on control trials which immediately preceded the test trials throughout the sessions. Bottom: Response to the single-tone stimulus on test trials in which the second stimulus was omitted.

\section{DISCUSSION}

Results of the present study reveal an association between the magnitude of the cardiac orienting response and the level of behavioral competence in developmentally disabled infants and young children. Specifically, the magnitude of cardiac deceleration to simple nonsignal stimuli was inversely correlated with adaptive behavior scores on the ABSI. The stimulus-induced changes in heart rate appeared to reflect the orienting response, since they (1) were evoked by novel stimuli, (2) demonstrated habituation over trials, (3) were subject to dishabituation on a change in the stimulus, and (4) were predominantly deceleratory (Graham, 1979). In contrast to the cardiac OR, cardiac measures of habituation and simple associative learning were not predictive of the level of adaptive function.
The observed relationship between the magnitude of the cardiac OR and ABSI scores was largely independent of age and baseline heart rate. Cardiac responses to the single stimuli of the inventory sessions and to the initial single-tone stimulus presented in the associative training sessions consistently exhibited a relationship to adaptive functioning, but were uncorrelated with either age or baseline heart rate. Similarly, reactivity to the paired conditioning stimuli was correlated with an age-independent component of the ABSI variance, as indicated by its significant partial correlation with ABSI scores. Cardiac reactivity to the paired conditioning stimuli, however, was also partially intercorrelated with age and with baseline heart rate. It remains unclear why these intercorrelations were observed only with the paired stimuli.

Neither the locus nor the mechanisms of the relationship between cardiac reactivity and adaptive behavior are 
readily apparent. The present findings are in accord with the studies of Karrer and Clausen (1964) and Johnson (1976), which demonstrated a tendency toward increased cardiac deceleration to nonsignal stimuli in mentally retarded subjects. Other studies, however, either have reported no consistent differences in the cardiac responses of retarded and normal subjects (Clausen et al., 1976; Powazek \& Johnson, 1973) or have shown the opposite pattern (Bradley-Johnson \& Travers, 1979). Similarly, no simple pattern of results has emerged from GSR studies of mentally retarded subjects (see review by Stern \& Janes, 1973). Procedural details differ considerably from study to study, however, and variables such as stimulus intensity (Johnson, 1976), signal value of the stimuli (Bradley-Johnson \& Travers, 1979; Powazek \& Johnson, 1973), and characteristics of the subject populations (Clausen et al., 1976; James \& Barry, 1980; Vogel, 1961) can dramatically influence the pattern of results. In contrast to this literature, results of the present study demonstrated a relationship between cardiac response magnitude and the level of adaptive behavior which appeared consistently across experimental conditions and was characteristic of all subject groups. Four unique features of the present study may have contributed to this outcome. First, subjects of the present study were considerably younger than those of previous studies. Second, the range and severity of the developmental disabilities of the present subjects were appreciably greater than those typically examined. Third, unlike most previous studies, we employed a vibrotactile stimulus which yielded a much higher relationship between cardiac responses and adaptive behavior than did an auditory stimulus. Finally, we used a measure of adaptive behavior as the criterion, which may have provided a broader sensitivity to multiple dimensions of functional competence.

The failure of simple learning phenomena to differentiate among varying levels of behavioral competence in the present study indicates that the habituation-omission paradigm is either tapping capacities too fundamental to be discriminatory or reflecting processes that are not consistently related to the origins of developmental disabilities (Goldstein \& Oakley, 1985). These results are consistent with the experimental literature on older retarded populations. In general, studies of the acquisition of simple classically conditioned responses have not revealed notable deficits in retarded populations, except when trace stimuli or long interstimulus intervals are employed (Grings, Lockhardt, \& Dameron, 1962; Ross \& Ross, 1976). Similarly, the omission response to CS-alone test trials during conventional GSR conditioning also fails to differentiate between normal and developmentally disabled subjects (Grings et al., 1962). The literature on habituation processes is less clear, and there is a growing appreciation in the literature of the distinction between the $\mathrm{OR}$ as an index to novelty and that related to stimulus significance (Barry, 1982; Johnson, 1976; Maltzman \& Langdon, 1982). Conflicting results may relate in part to whether adaptively significant or nonsignal stimuli are employed. Retarded subjects often show normal habituation to salient nonsignal stimuli, such as those used in the present study, although they may habituate more quickly than normal subjects to weak or task-relevant stimuli (Johnson, 1976; Karrer et al., 1979; Luria, 1963).

In summary, the level of adaptive function of developmentally disabled infants and young children was not reflected in cardiac measures of habituation or simple associative learning. Rather, it was the unique ageindependent component of cardiac reactivity that clearly delineated the subjects with respect to the level of adaptive competence, with more impaired subjects displaying larger deceleratory orienting responses. Whether this pattern of reactivity is a consequence or an integral determinant of the level of adaptive behavior is not clear. The potentially interfering influence of exaggerated orienting, with its descriptive origins in the concept of external inhibition (Pavlov, 1927), is consistent with the characteristic appearance of attentional deficits in developmental disabilities (Luria, 1963; Zeaman \& House, 1963). In contrast, efforts to document the contribution of the OR to learning process in normal subjects are limited, and the results inconclusive (Cousins, 1976; Putnam, Ross, \& Graham, 1974).

The present results are thus consistent with the view that attentional deficits in developmentally disabled children may result, at least in part, from an exaggerated and persistent pattern of reactivity to nonsignificant stimuli (Luria, 1963). In the subjects of the present study, this excessive reactivity did not arise from a loss of habituation processes. Rather, the more impaired subjects showed an enhanced cardiac reactivity that was apparent both on the initial stimulus presentations and on subsequent trials after habituation. It is unlikely, however, that a single dimension of reactivity can adequately account for the broad range of deficits manifested by developmentally impaired children. The exaggerated responding among the children with $\mathrm{CP}$, a disability characterized by deficits in motoric function which may or may not be accompanied by cognitive deficits, raises the possibility that the dimension along which the subjects were ordered is one of motor competence. Thus, although there may exist a correspondence between adaptive behavior and motor functions in these children, these capacities may be dissociated from cognitive processes. Adaptive behavior, however, is highly correlated with conventional measures of intelligence among less impaired, older populations (Ellis, Harris, \& Barker, 1983), and cognitive competence must ultimately depend to a significant extent upon sensorimotor integrity. Nevertheless, in the absence of independent verification of the level of cognitive function in subjects immune to conventional assessment, the understanding of this relationship must remain speculative. The use of psychophysiological measures and creatively applied behavioral paradigms may provide a means to address this issue. 


\section{REFERENCES}

BARRY, R. J. (1982). Novelty and significance effects in the fractionation of phasic OR measures: A synthesis with traditional OR theory. Psychophysiology,19, 28-35.

Berntson, G. G., Tuber, D. S., Ronca, A. E., \& Bachman, D. S. (1983). The decerebrate human: Associative learning. Experimental Neurology, 81, 77-88.

Bradley-Johnson, S., \& Travers, R. M. W. (1979). Cardiac change of retarded and nonretarded infants to an auditory signal. American Journal of Mental Deficiency, 83, 631-636.

Clausen, J., Lidsky, A., \& Serson, E. A. (1976). Measurements of autonomic functions in mental deficiency. In R. Karrer (Ed.), Developmental psychophysiology of mental retardation: Concepts and studies. Springfield: Thomas.

Cousins, L. R. (1976). Individual differences in the orienting reflex and children's discrimination learning. Psychophysiology,13, 479-487.

D'Eugenio, D. B., \& MOERSCH, M. S. (1981). Developmental programming for infants and young children: Preschool assessment and application. Ann Arbor: University of Michigan Press.

Ellis, N. R., HARrIs, L. A., \& BarKer, H. R. (1983). Ecological validity of laboratory-type discrimination-learning tasks. American Journal of Mental Deficiency, 88, 106-108.

Fox, N. A., \& LewIS, M. (1983). Cardiac response to speech sounds in preterm infants: Effects of postnatal illness at three months. Psychophysiology,20, 481-488.

Goldstein, L. H., \& OAKLEY, D. A. (1985). Expected and actual behavioral capacity after diffuse reduction in cerebral cortex: Suggestions for rehabilitative techniques with the mentally handicapped and head injured. British Journal of Clinical Psychology, 24, 13-24.

GRAHAM, F. K. (1979). Distinguishing among orienting, defensive, and startle reflexes. In H. D. Kimmel, E. H. van Olst, \& J. F. Orlebeke (Eds.), The orienting reflex in humans. Hillsdale, NJ: Erlbaum.

Grings, W. W. (1960). Preparatory set variables related to classical conditioning of autonomic responses. Psychological Review, 67, 243-252.

Grings, W. W., Lockart, R. A., \& Dameron, L. E. (1962). Conditioning autonomic responses of mentally subnormal individuals. Psychological Monographs, 76(39, Whole No. 558).

JAMES, A. L., \& BARRY, R. J. (1980). Respiratory and vascular responses to simple stimuli in autistics, retardates and normals. Psychophysiology, 17, 541-547.

JoHNSON, J. T. (1976). The orienting reflex and mental retardation. In R. Karrer (Ed.), Developmental psychophysiology of mental retardation: Concepts and studies. Springfield: Thomas.

Karrer, R., \& Clausen, J. (1964). A comparison of mentally deficient and normal individuals upon four dimensions of autonomic activity. Journal of Mental Deficiency Research, 8, 149-163.

Karrer, R., Nelson, M. N., \& Galbraith, G. C. (1979). Psychophysiological research with the mentally retarded. In N. R. Ellis (Ed.), Handbook of mental deficiency: Psychological theory and research. Hillsdale, NJ: Erlbaum.

Krafchuk, E. E., Tronick, E. Z., \& Clifton, R. K. (1983). Behavioral and cardiac responses to sound in preterm neonates varying in risk status: A hypothesis of their paradoxical reactivity. In R. Kearsley \& I. Sigel (Eds.), Infants at risk: Assessment of cognitive function. Hillsdale, NJ: Erlbaum.

Leland, H. (1982). Assessment of adaptive behavior. In K. D. Paget \& B. A. Bracken (Eds.), Psychoeducational assessment of preschool children. New York: Grune \& Stratton.

Leland, H., Shoaee, M., McElwain, D., \& Christie, R. (1981). Adaptive Behavior Scale for Infants and Early Childhood. Columbus: Ohio State University Press.

LURIA, A. R. (1963). The mentally retarded child. New York: Macmillan.

Maltzman, I., \& LANGDon, B. (1982). Novelty and significance as determiners of the GSR index of the orienting reflex. Physiological Psychology, 10, 229-234.

NiHira, K., Foster, R., ShellhaAs, M., \& Leland, H. (1974). AAMD adaptive behavior scale. Washington: American Association of Mental Deficiency.

Pavlov, I. P. (1927). Conditioned reflexes. London: Oxford University Press.

Porges, S. W. (1983). Heart rate patterns in neonates: A potential diagnostic window to the brain. In T. Field \& A. Sostek (Eds.), In fants born at risk: Physiological, perceptual, and cognitive processes. New York: Grune \& Stratton.

Powazek, M., \& Johnson, J. T. (1973). Heart rate response to novel and signal stimuli in nonretarded and retarded subjects. American Journal of Mental Deficiency, 78, 286-291.

Putnam, L. E., Ross, L. E., \& Graham, F. K. (1974). Cardiac orienting during "good" and "poor" differential eyelid conditioning. Journal of Experimental Psychology, 102, 563-573.

Ross, S. M., \& Ross, L. E. (1976). The conditioning of skeletal and autonomic responses: Normal-retardate stimulus trace differences. In N. R. Ellis (Ed.), International review of research in mental retardation. New York: Academic Press.

Sokolov, Y. N. (1963). Perception and the conditioned reflex. New York: Pergamon.

Stern, J. A., \& JANes, C. L. (1973). Personality and psychopathology. In W. F. Prokasy \& D. C. Raskin (Eds.), Electrodermal activity in psychological research. New York: Academic Press.

Tuber, D. S., Berntson, G. G., Bachman, D. S., \& Allen, J. N. (1980). Associative learning in premature hydranencephalic and normal twins. Science, 210, 1035-1037.

VoGEL, W. (1961). The relationship of age and intelligence to autonomic functioning. Journal of Comparative \& Physiological Psychology, 54, 133-138.

ZEAMAN, W., \& House, B. J. (1963). The role of attention in retardate discrimination learning. In N. R. Ellis (Ed.), Handbook of mental deficiency. New York: McGraw-Hill.

(Manuscript received March 28, 1985; revision accepted for publication September 1, 1985.) 